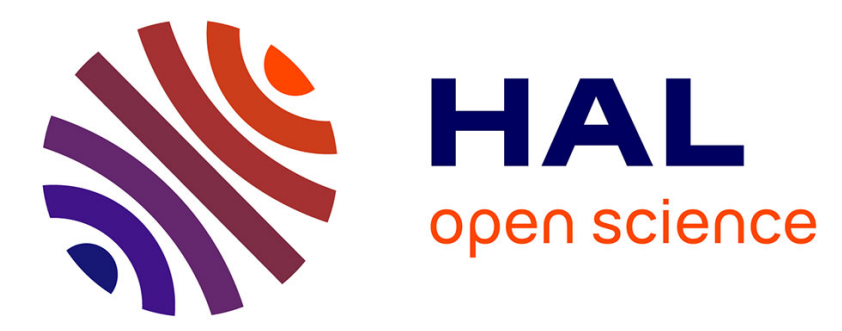

\title{
Ferrimagnetism in Diluted Mixed Spin-1/2 and Spin-3/2 Ising Systems
}

\author{
A. Bobák, M. Jurcisin
}

\section{To cite this version:}

A. Bobák, M. Jurcisin. Ferrimagnetism in Diluted Mixed Spin-1/2 and Spin-3/2 Ising Systems. Journal de Physique IV Proceedings, 1997, 07 (C1), pp.C1-179-C1-180. 10.1051/jp4:1997165 . jpa00255112

\section{HAL Id: jpa-00255112 https://hal.science/jpa-00255112}

Submitted on 1 Jan 1997

HAL is a multi-disciplinary open access archive for the deposit and dissemination of scientific research documents, whether they are published or not. The documents may come from teaching and research institutions in France or abroad, or from public or private research centers.
L'archive ouverte pluridisciplinaire HAL, est destinée au dépôt et à la diffusion de documents scientifiques de niveau recherche, publiés ou non, émanant des établissements d'enseignement et de recherche français ou étrangers, des laboratoires publics ou privés. 


\title{
Ferrimagnetism in Diluted Mixed Spin-1/2 and Spin-3/2 Ising Systems
}

\author{
A. Bobák and M. Jurcisin \\ Department of Theoretical Physics and Geophysics, Faculty of Natural Sciences, P.J. Safárik University, \\ Moyzesova 16, 04154 Kosice, Slovakia
}

\begin{abstract}
Using an effective field theory with correlations we study a diluted mixed spin-1/2 and spin-3/2 Ising ferrimagnetic system with a crystal field interaction $D(D<0)$ on a honeycomb lattice. We find that the system may exhibit two compensation points in the total magnetization curve, although the corresponding system in the absence of dilution or with $D=0$ never exhibits any compensation point at $T \neq 0 K$.
\end{abstract}

\section{INTRODUCTION}

Ferrimagnetism has been intensively investigated in the past both experimentally and theoretically. From a theoretical point of view, the two-sublattice mixed-spin Ising system consisting of spins $s_{A}=1 / 2$ and $s_{B}\left(s_{B}>1 / 2\right)$ has been introduced as a simple model showing a certain type of ferrimagnetism. In particular, it was shown for the case $s_{B}=1$ that this model with site-dilution on a honeycomb lattice may exhibit two compensation points [1], although the same system consisting of $s_{A}=1 / 2$ and $s_{B}=3 / 2$ never exhibits any compensation point [2].

On the other hand, for Ising systems with $s_{B}>1 / 2$, one has to expect an appreciable crystal-field splitting of the energy levels, which could strongly influence the physical properties of the system. The purpose of this work is thus to clarify the effects of crystal field interaction on a compensation temperature in the diluted mixed spin Ising ferrimagnetic system when the values of $s_{A}$ and $s_{B}$ are selected as $s_{A}=1 / 2$ and $s_{B}=3 / 2$ on a honeycomb lattice.

\section{FORMULATION}

We consider a site-diluted mixed spin-1/2 and spin-3/2 Ising ferrimagnetic system in the presence of a crystal field interaction $D$ described by the Hamiltonian

$$
H=-J \sum_{\langle i, j\rangle} s_{i}^{A} s_{j}^{B} \xi_{i}^{A} \xi_{j}^{B}-D \sum_{j} \xi_{j}^{B}\left(s_{j}^{B}\right)^{2}
$$

where $s_{i}^{A}$ takes, on sublattice $A$, the values $\pm 1 / 2$, and $s_{j}^{B}$ on sublattice $B$, can be $\pm 1 / 2$ and $\pm 3 / 2 . J(J<0)$ is the exchange interaction and $\xi_{i}^{A}\left(\xi_{j}^{B}\right)$ is the site occupancy number which takes the value of unity or zero, depending on whether the site $i(j)$ is occupied by a magnetic atom of type $A(B)$ or not.

To evaluate the averaged sublattice magnetizations $m_{A}, m_{B}$ and the parameters $q$, $r$, defined by

$$
m_{A}=<\xi_{i}^{A}<s_{i}^{A}>_{c}, \quad m_{B}=<\xi_{j}^{B}<s_{j}^{B}>_{c}, \quad q=<\xi_{j}^{B}<\left(s_{j}^{B}\right)^{2}>_{c}, \quad r=<\xi_{j}^{B}<\left(s_{j}^{B}\right)^{3}>_{c},
$$

where $\langle\cdots\rangle\left(\langle\cdots\rangle_{c}\right)$ denotes the thermal (configurational) average, the effective-field theory with correlation [3] can be applied to this problem. By using a similar calculation as in $[1,3]$, one finds

$$
\begin{gathered}
m_{A}=\left.p_{A}\left\{1+p_{B}\left[K_{0}(\gamma)-1\right]+m_{B} K_{1}(\gamma)+q K_{2}(\gamma)+r K_{3}(\gamma)\right\}^{z} f(x)\right|_{x=0}, \\
<\xi_{j}^{B}<\left(s_{j}^{B}\right)^{k}>>_{c}=\left.p_{B}\left\{1+p_{A}[\cosh (\gamma / 2)-1]+2 m_{A} \sinh (\gamma / 2)\right\}^{z} F_{k}(x)\right|_{x=0} \quad(k=1,2,3),
\end{gathered}
$$

where $p_{\alpha}=\left\langle\xi_{i}^{\alpha}\right\rangle_{c}$ is the concentration of magnetic $\alpha$ atoms $(\alpha=A$ or $B$ ), $z$ is the coordination number and

$$
K_{0}(\gamma)=\frac{1}{8}[9 \cosh (\gamma / 2)-\cosh (3 \gamma / 2)], \quad K_{1}(\gamma)=\frac{1}{12}[27 \sinh (\gamma / 2)-\sinh (3 \gamma / 2)],
$$




$$
K_{2}(\gamma)=\frac{1}{2}[\cosh (3 \gamma / 2)-\cosh (\gamma / 2)], \quad K_{3}(\gamma)=\frac{1}{3}[\sinh (3 \gamma / 2)-3 \sinh (\gamma / 2)],
$$

with $\gamma=J \nabla$ and $\nabla=\partial / \partial x$ is the differential operator. The functions $f(x)$ and $F_{k}(x)$ are given by

$$
\begin{gathered}
f(x)=\frac{1}{2} \tanh (\beta x / 2), \quad F_{1}(x)=\frac{1}{2} \frac{3 \sinh (3 \beta x / 2)+\exp (-2 D \beta) \sinh (\beta x / 2)}{\cosh (3 \beta x / 2)+\exp (-2 D \beta) \cosh (\beta x / 2)}, \\
F_{2}(x)=\frac{1}{4} \frac{9 \cosh (3 \beta x / 2)+\exp (-2 D \beta) \cosh (\beta x / 2)}{\cosh (3 \beta x / 2)+\exp (-2 D \beta) \cosh (\beta x / 2)}, \quad F_{3}(x)=\frac{1}{8} \frac{27 \sinh (3 \beta x / 2)+\exp (-2 D \beta) \sinh (\beta x / 2)}{\cosh (3 \beta x / 2)+\exp (-2 D \beta) \cosh (\beta x / 2)},
\end{gathered}
$$

where $\beta=1 / k_{B} T$. The averaged total magnetization per site is then given by

$$
M=\frac{1}{2}\left(m_{A}+m_{B}\right) \text {. }
$$

The second-order line is then given by substituting equations (4) in (3) and linearizing the latter. On the other hand, the compensation temperature $T_{k}\left(T_{k}<T_{c}\right)$, at which the total magnetization vanishes, can be evaluated by requiring the condition $m_{B}=-m_{A}$ in the coupled equations (3) and (4).

\section{RESULTS AND DISCUSSIONS}

Figure 1 a) shows some typical phase diagrams in the $\left(T, p_{B}\right)$ plane for the ferrimagnet witl $z=3$ and $p_{A}=1$, when the value of $D /|J|$ is changed. The solid and dashed lines represent the variations of the compensation temperature $T_{k}$ and transition temperature $T_{c}$, respectively. The results clearly show that the compensation point at $T \neq 0 K$ may exist in the system with $p_{A}=1$ and $p_{B}<1$ for an appropriate negative $D$ value. Notice that the curve labelled $D /|J|=-0.4$ exhibits even two compensation points, although this is not sufficiently extensive to be seen on the scale of figure $1 \mathrm{a}$ ). The appearence of the compensation point (or points) in this system comes from both the dilution of the sublattice $B$ and the negative crystal field interaction. Indeed, the corresponding system in the absence of dilution $\left(p_{A}=p_{B}=1\right)[4]$ or with $D=0[2]$ does not exhibit, any compensation point at $T \neq 0 \mathrm{~K}$.

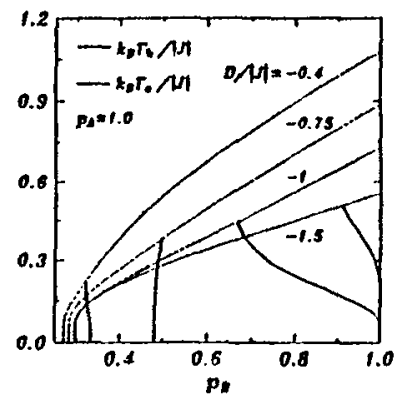

a)

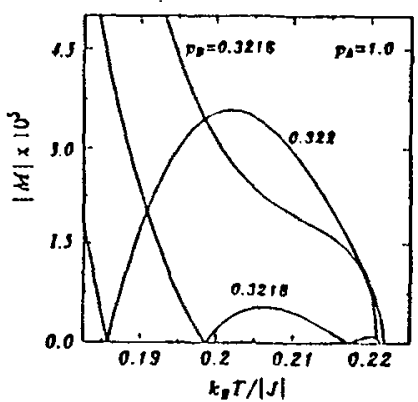

b)

Figure 1: a) Transition temperature $T_{c}$ (dashed lines) and compensation temperature $T_{k}$ (solid lines) versus the concentration of agnetic atoms $\left(p_{B}\right)$ in the sublattice $B$ for the system with $z=3$ and $p_{A}=1$, when the value of $D /|J|$ is clianged. b) $M-T$ curves for the system with $p_{A}=1$ and $D /|J|=-0.4$, when $p_{B}$ is changed,

In order to confirm the prediction of two compensation points, let us examine the total magnetization $|M|$ as a function $T$ especially for the system with $p_{A}=1$ and $D / \mid H=-0.4$, when the value of $p_{B}$ is changed. As socn from figure $(\mathrm{b})$, the $M-T$ curve labelled $p_{B}=0.322$ shows only one compensation point, however, the curve with $p_{B}=0.3218$ exhibits two compensation points. We hope that the results obtained in this work may be helpful when the experimental data of ferrimagnetic materials are analysed.

\section{References}

[1] Bobák A. and Jaščur M., Phys.Rev. B 51 (1995) 11533-11537.

[2] Bobák A. and Jurčišin M., J.Magn.Magn.Mater. (in press).

[3] Kaneyoshi T., Tucker J. W. and Jaščur M., Physica A 186 (1992) 495-512.

[4] Kaneyoshi T., Jaščur M. and Tomczak P., J.Phys. : Condens.Matter 4 (1992) L653-L658. 